\section{Jean-Paul Lévy}

\section{Traitements du SIDA : recherche de nouveaux médicaments et élaboration de thérapies géniques}

Tout l'arsenal des techniques qui permettent d'élaborer de nouveaux médicaments est aujourd'hui mis en ouvre pour mettre au point une thérapeutique antivirale efficace sur le virus HIV-1. C'est par une approche classique que fut découvert l'AZT, seule molécule d'utilisation médicale courante, mais dont l'efficacité limitée fait rechercher des dérivés plus actifs et moins toxiques. La modélisation moléculaire d'inhibiteurs, réalisée à partir d'une structure protéique cible connue, constitue une approche qui pourrait se révéler extrêmement puissante. Il demeure qu'en dépit de nouvelles pistes prometteuses, la synthèse de molécules antivirales complètement efficaces risque de ne pas être rapide. L'importance de l'enjeu justifie donc d'analyser les potentialités offertes par des stratégies moins classiques, telle la thérapie génique somatique par immunisation intracellulaire ou toxigénétique, qui pourrait être appliquée en complément des autres approches thérapeutiques.

\section{ADRESSE}

J.-P. Lévy : professeur d'hématologie. Hôpital Cochin. Directeur de l'ANRS. Directeur de

l'Institut Cochin de Génétique Moléculaire,
22, rue Méchain, 75014 Paris, France. a seule thérapeutique antiHIV d'utilisation médicale courante repose actuellement sur l'administration de médicaments inhibiteurs de l'infection virale, dont le chef de file est la zidovudine (AZT) [1]. L'activité antivirale de ce produit a été identifiée par la méthode dont procède classiquement la découverte de médicaments antiviraux : le criblage systématique de molécules, à la recherche d'une activité inhibant l'infection virale de cellules en culture [2]. Des centaines de milliers de pro- duits dont peuvent disposer les laboratoires et industries pharmaceutiques, ainsi que des préparations d'extraits végétaux ou de fermentation ont ainsi fait l'objet de tests au hasard. Certes le rendement d'une telle méthode est très faible mais elle peut mener à la découverte de séries moléculaires nouvelles, actives sur n'importe quel stade de la réplication virale. Cette stratégie peut être complétée par la recherche à partir d'un produit d'efficacité connue, de molécules plus efficaces et moins toxiques [3]. Théoriquement d'un meilleur 
rendement, cette méthode a donné lieu à l'étude de milliers d'analogues nucléosidiques dérivés de l'AZT, de la didéoxyinosine (ddI) ou de la didéoxycytosine (ddC), molécules inhibitrices de la transcriptase inverse virale. Cette recherche est cependant handicapée par le peu d'informations dont nous disposons sur les raisons pour lesquelles telle ou telle molécule est efficace ou toxique. Une troisième stratégie pour isoler de nouveaux médicaments, probablement la plus prometteuse pour l'avenir mais encore relativement peu employée en pharmacologie, est la modélisation moléculaire ou molecular design. Elle consiste, une fois la structure tridimensionnelle de la molécule cible établie et ses zones fonctionnelles identifiées, à dessiner un inhibiteur théorique capable d'effectuer avec la cible une liaison stable aboutissant à une inhibition irréversible, quel qu'en soit le mécanisme. Appliquée à la recherche de médicaments anti-HIV, cette méthode qui vient de donner des résultats spectaculaires pour la mise au point, au moins in vitro, d'inhibiteurs de protéase [4-5], nécessite l'identification des diverses cibles potentielles.

\section{Les cibles possibles d'un traitement anti-HIV}

Le cycle des rétrovirus est bien connu (figure $1, p .832$ ) et a fait l'objet de nombreuses revues [6-9]. Le virus, fusionnant son enveloppe avec la membrane cellulaire, injecte dans le cytosol, la totalité de sa capside. A l'intérieur de celle-ci, la transcription inverse est assurée par la transcriptase inverse du virus, aboutissant à la synthèse d'un $\mathrm{ADN}$ proviral double brin, puis au transport de cet $\mathrm{ADN}$ lié aux protéines capsidiales dans le noyau cellulaire. Là, une endonucléase ou "intégrase " permet son intégration dans le génome cellulaire. Intervient alors une phase de latence plus ou moins prolongée, fonction de conditions encore mal connues et au cours de laquelle il n'y a pas de traduction du génome viral. A terme, le cycle viral se poursuit par la traduction de son génome (figure 2, p. 833) conduisant à la production d'ARN viraux qui vont jouer d'une part le rôle de $\mathrm{m} / \mathrm{s} \mathrm{n}^{\circ} 8$, vol. 7, octobre 91 génome pour les futures particules filles et, d'autre part, celui d'ARN messagers pour la synthèse des protéines virales. Deux protéines jouent un rôle tout à fait particulier à ce stade : Tat et Rev [10]. Tat, codée par l'un des messagers les plus précoces du virus est une protéine transactivatrice qui semble intervenir à la fois dans la transcription et dans la régulation post-transcriptionnelle de l'expression virale. Rev, synthétisée comme Tat grâce à certains des petits messagers initiaux du virus, assure de son côté le passage cytoplasmique des grands messagers qui vont aboutir à la synthèse des protéines structurales. Après la synthèse des constituants protéiques internes sous forme de longs précurseurs non clivés, la particule virale bourgeonne à la membrane cellulaire dans laquelle viennent s'enclaver ses glycoprotéines de surface (gp120) et transmembranaire (gp41). La maturation complète du virion ne s'achève qu'après la séparation de la particule virale de la membrane cellulaire, grâce à l'activité de la protéase virale qui clive les grands précurseurs des protéines internes. Cela permet la mise en place des trois protéines issues du gène gag (protéines de la matrice qui sous-tend l'enveloppe virale, de la capside interne et de la nucléocapside associée à l'ARN), ainsi que des enzymes issues du gène pol, indispensables à la réplication (la transcriptase inverse, l'intégrase et la protéase elle-même). A côté de ces données relativement claires, on sait que d'autres protéines ( $\mathrm{Vpu}, \mathrm{Vpr}$, Vif, Nef), interviennent dans le cycle viral, mais leur rôle, surtout en ce qui concerne Vif et Nef, est moins bien connu. Par conséquent, elles ne peuvent représenter pour le moment que des cibles très incertaines de médicaments antiviraux.

Les cibles potentielles étant identifiées, on peut en principe concevoir trois types de médicaments antiviraux. (1) Ceux qui bloquent l'infection de la cellule à quelque stade que ce soit, entre l'interaction du virus et de son récepteur à la surface cellulaire, et l'intégration du génome. (2) Ceux qui, à un stade ultérieur, empêchent la cellule infectée de produire des particules virales ou ne laissent produire que des particules inef- ficaces. De tels médicaments pourraient agir à n'importe quelle étape entre la transcription et la maturation complète du virion après sa sortie de la cellule. (3) Ceux enfin qui, faisant la part du feu, détruiraient électivement les cellules infectées, réduisant ainsi le risque d'extension de l'infection virale à d'autres cellules.

\section{Médicaments inhibiteurs de I'infection virale}

Comme nous l'avons déjà mentionné, la famille des nucléosides inhibiteurs de la transcriptase inverse (AZT, ddI, ddC), inclut tous les médicaments qui sont de pratique médicale courante dans le traitement de l'infection par le HIV. De nombreux analogues des mêmes médicaments sont actuellement à l'étude sans qu'à ce jour ait été identifiée une molécule à la fois plus active et dépourvue de toxicité médullaire ou neurologique. Leur mode d'action est l'inhibition de la transcription inverse, mais la part respective d'une inhibition directe de l'enzyme et d'un arrêt d'élongation des chaînes reste imprécise. Les molécules de la famille des TIBO, dérivés de benzodiazépines dépourvues d'action sur le système nerveux central et dont certaines sont, in vitro, de puissants inhibiteurs de la réplication du HIV-1 (mais non du HIV-2), seraient également des inhibiteurs de la transcriptase inverse $[11,12]$. Pour l'heure, les essais cliniques sur l'homme, des premiers TIBO sont plutôt décevants, mais l'étude de cette famille moléculaire se poursuit.

En aval de la transcription inverse et avant l'intégration, on peut imaginer d'autres cibles de médicaments antiviraux, mais tout reste encore à faire en ce domaine peu exploré. L'inhibition de la ribonucléase $\mathrm{H}$, enzyme nécessaire à la synthèse du deuxième brin d'ADN au cours de la transcription inverse, est une piste potentielle. L'intégrase pourrait aussi, en principe, constituer une cible importante, mais cette enzyme virale est encore mal connue [13]. Quant au rôle d'éventuelles protéines cellulaires de transport interagissant avec des constituants de la capside ou d'autres constituants viraux pour assurer le transfert du complexe acide nucléi- 
Figure 1. Cycle du virus HIV-1 et cibles potentielles d'une thérapie anti-HIV-1. A. Étapes précédant I'intégration virale dans le génome. 1 : le virus reconnaît la molécule CD4 et se fixe à la cellule. Les CD4 solubles, des polysaccharides ou des anticorps dirigés contre des protéines de l'enveloppe virale peuvent empêcher la reconnaissance et/ou la fixation du virus à la cellule cible. 2 : après fusion des membranes virales et cellulaires, la capside pénètre dans le cytoplasme. 3 : à l'intérieur de la capside s'effectue la synthèse de l'ADN proviral par transcription inverse de l'ARN génomique à l'aide de la transcriptase inverse virale. Cette étape peut être bloquée par les inhibiteurs de la transcriptase inverse: $A Z T$, ddl, ddC, etc. 4 : I'ADN proviral pénètre dans le noyau et 5 : s'intègre au chromosome de l'hôte grâce à l'action d'une intégrase virale. On ne dispose pas à l'heure actuelle d'inhibiteurs d'intégrase. B. Étapes postérieures à l'intégration virale. 6 : la transcription du provirus, stimulée par la protéine Tat, conduit à la formation d'un ARN de 35S (ARN génomique). Des inhibiteurs de Tat ou des leurres la détournant de son activité normale pourraient bloquer la transcription du virus. 7 : la maturation de l'ARN viral, le transfert vers le cytoplasme et l'équilibre entre les différents ARN sont sous le contrôle de la protéine Rev. On ne connaît pas d'inhibiteurs de Rev mais des leurres constitués de la séquence reconnue par Rev (RRE, Rev responsive element), pourraient la détourner de son activité normale. 8 : traduction des diverses protéines virales. Les messagers viraux pourraient être détruits par des ribozymes spécifiques ou inactivés par des ARN antisens. 9 : la maturation des protéines virales comporte des étapes de glycosylation et myristylation qui pourraient être inhibées, rendant les protéines inaptes a former un virion fonctionnel. 10 : dimérisation de l'ARN génomique viral. 11 : I'assemblage et le bourgeonnement des virions pourraient être l'étape cible de l'interféron. 12 : la maturation des virions est sous l'action de la protéase virale. La recherche $d^{\prime}$ inhibiteurs de protéase est activement engagée.
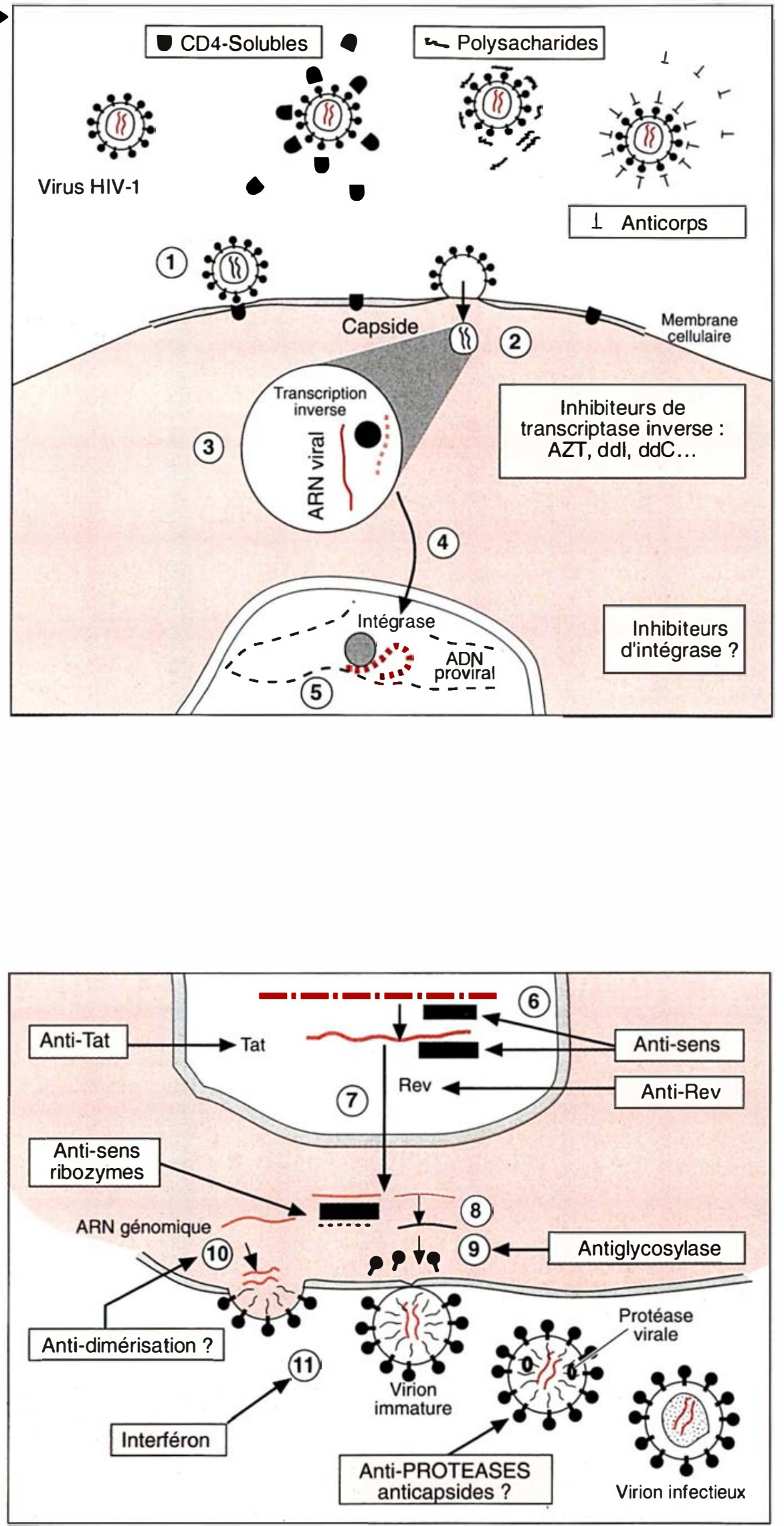


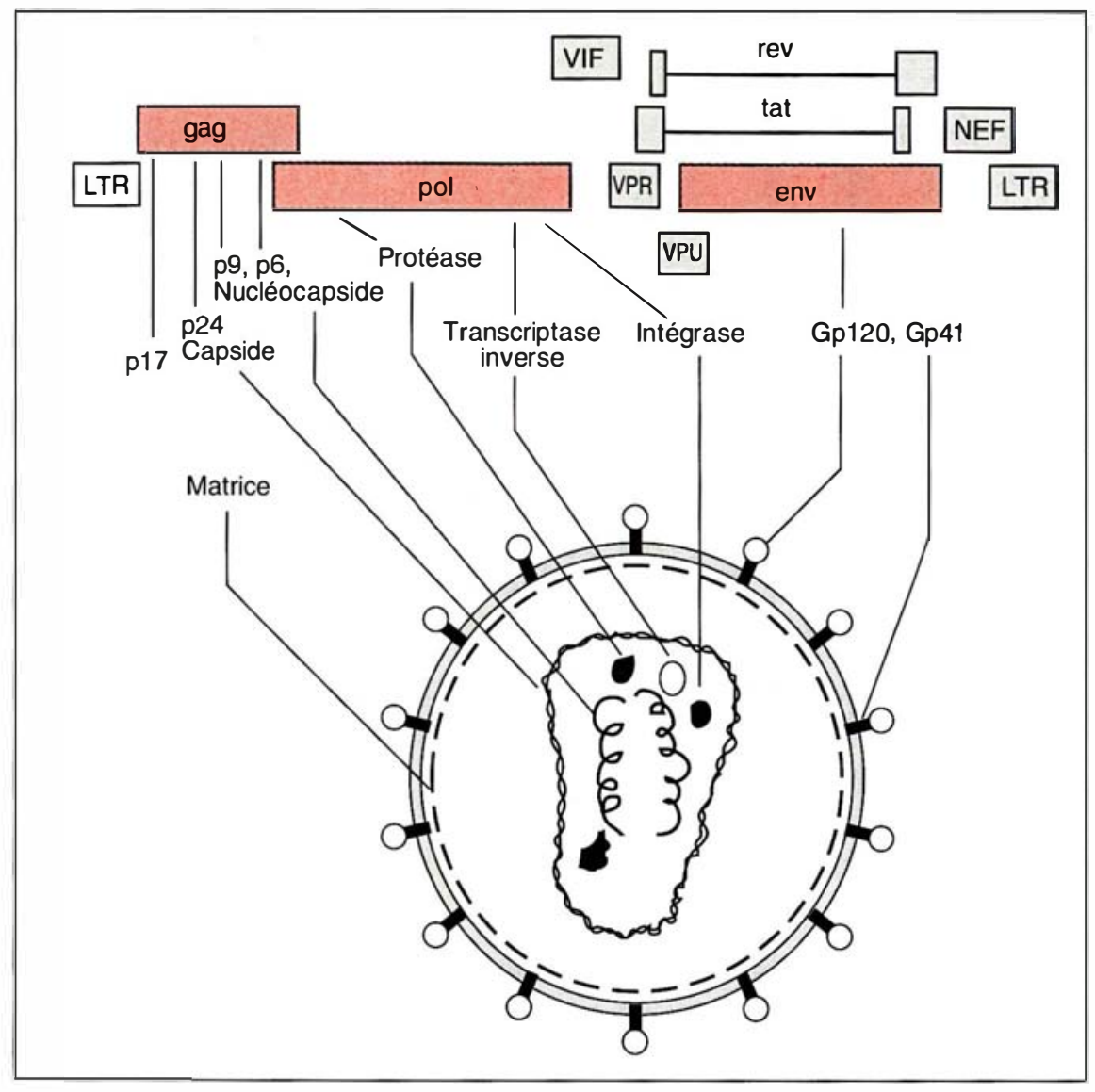

Figure 2. Le génome du virus HIV-1 et la particule virale. Les gènes de régulation sont en grisé. LTR: long terminal repeat, longue répétition terminale.

que/intégrase, du cytoplasme vers le noyau, il reste pour le moment incertain et donc très en aval de nos possibilités d'intervention.

En revanche, en amont de la transcription inverse, de multiples pistes sont actuellement explorées, dans le but d'aboutir à des médicaments inhibiteurs soit de l'adhérence du virus à son récepteur, soit de la fusion de l'enveloppe virale et de la membrane cellulaire. Dans l'un et l'autre cas, l'infection serait bloquée avant la pénétration du virus dans la cellule. Trois groupes de molécules intervenant à ces étapes font l'objet d'études intensives sans que pour autant on puisse envisager à brève échéance une utilisation à large échelle en clinique humaine.

Les anticorps neutralisants constituent l'un des moyens naturels de défense contre le virus. Qu'il s'agisse de protéines obtenues à partir du $\mathrm{m} / \mathrm{s} n^{\circ} 8$, vol. 7 , octobre 91 immédiatement après leur survenue accidentelle. D'une façon générale, l'utilisation prolongée se heurte aux obstacles habituels à l'emploi des anticorps en thérapeutique humaine : la nécessité d'injections répétées par voie veineuse, l'efficacité limitée de ces anticorps et l'immunisation secondaire aboutissant à la production d'anti-anticorps qui en inhibent les effets. Leur avenir thérapeutique semble donc limité en dehors de cas particuliers.

Les polysaccharides sulfatés ont un mécanisme d'action mal connu. On admet en général qu'ils interagissent de façon non spécifique avec l'enveloppe virale et bloquent sa capacité de réaction avec les récepteurs cellulaires, mais une action sur la cellule cible est également possible. Ces médicaments, dont le modèle initial a été le sulfate de dextran [15], ont une efficacité antivirale modérée exigeant d'importantes concentrations sanguines; ils doivent être injectés par voie veineuse et ont une activité anticoagulante de type héparine, ce qui en limite l'emploi. Les produits actuellement existant ne semblent pas destinés à un avenir thérapeutique notable, mais la poursuite des études autour de cette famille moléculaire est certainement justifiée.

Les molécules CD4 solubles. La molécule CD4 est le récepteur principal du virus à la surface des cellules hématopoïétiques. Il a donc été imaginé de préparer des molécules CD4 solubles, débarrassées de leur région transmembranaire d'ancrage cellulaire pour s'en servir de leurres destinés à bloquer les molécules gp120 à la surface du virus [16]. On espère ainsi empêcher l'accès du virion à la cellule. Il semble bien que l'efficacité de telles molécules soit faible et en fait surtout liée à leur capacité de séparer la protéine gp120 du virion. La durée de vie plasmatique réduite à quelques minutes et sa faible efficacité, ont fait que le CD4 soluble a dû être abandonné. D'autres molécules, dont la durée de vie peut aller jusqu'à 48 heures, ont été synthétisées en combinant les premiers domaines de la molécule CD4 à d'autres protéines telle l'albumine ou des régions Fc d'immunoglobulines, pour constituer alors ce que l'on 


\section{RÉFÉRENCES}

1. Mitsuya H, Weinbold KJ, Furman PA, et al. 3' Azido 3' deoxythymidine (BWA509U) : an antiviral agent that inhibits the infectivity and cytopathic effect of human $\mathrm{T}$ lymphoma virus type III/lymphadenopathy-associated virus in vitro. Proc Nall Acad Sci USA 1985; 82 : 7096-100.

2. Mitsuya H, Broder S. Strategies for antiviral therapy in AIDS. Nature $1987 ; 325$ : 773-8.

3. Nasr M, Litterst, McGowan J. Computer-assisted structure-activity correlations of dideoxynucleoside analogs as potential anti-HIV drugs. Antiviral Res 1990; 14 : 125-48.

4. Roberts NA, Martin JA, Kinchington D, et al. Rational design of peptide based HIV proteinase inhibitors. Science $1990 ; 248$ : $358-61$

5. Blundell TL, Lapatto R, Wilderspin AF, et al. The 3-D structure of HIV-1 proteinase and the design of antiviral agents for the treatment of AIDS. Trends Biochem Sci $1990 ; 15: 425-30$

6. Haseltine WA. Molecular biology of the human immunodeficiency virus type 1. $F A S E B$ J $1991 ; 5$ : 2349-60.

7. Cullen BR. Regulation of HIV-1 gene expression. FASEB J 1991 ; 5 : 2361-8.

8. Cann AJ, Karn J. Molecular biology of HIV : new insights into the virus life-cycle. AIDS 1989 ; 3 (suppl 1) : S19-34.

9. Greene WC. The molecular biology of human immunodeficiency virus type 1 infection. New EnglJ Med 1991; 324: 308-16.

10. Rosen CA, Pavlakis GN. Tat and Rev : positive regulators of HIV gene expression. AIDS $1990 ; 4$ : 499-509.

11. Pauwels R, Andries K, Desmyter J, et al. Potent and selective inhibitor of HIV-1 replication in vitro by a novel series of TIBO derivatives. Science $1990 ; 343: 470-4$.

12. Merluzzi VJ, Hargrave KD, Labadia $M$, et al. Inhibition of HIV-1 replication by a non-nucleoside reverse transcriptase inhibitor. Science 1990; $250: 1411-3$.

13. Bushman F, Craigie R. Activities of human immunodeficiency virus (HIV) integration protein in vitro: specific cleavage and integration of HIV DNA. Proc Nall appelle une immunoadhésine [17-19]. L'efficacité in vitro de ces dérivés du CD4 soluble est claire et leur toxicité in vivo paraît faible malgré les craintes que l'on a pu avoir initialement d'un effet immunosuppresseur par interaction de ces molécules avec les molécules HLA de classe II des cellules immunocompétentes. L'obstacle principal à leur emploi est cependant la très faible activité antivirale observée in vivo, même à des concentrations élevées. Pour cette raison, malgré les espoirs initialement mis en eux, il est peu probable que les médicaments de cette famille puissent constituer, au moins à brève échéance, des médicaments importants dans le traitement de l'inf ection par le HIV. Toutefois les travaux se poursuivent pour tenter d'améliorer ce système particulièrement intelligent.

Médicaments susceptibles de bloquer la production de particules virales infectieuses par les cellules infectées

Les inhibiteurs de protéines virales Cinq protéines au moins constituent les cibles possibles d'une action antivirale postérieure à l'intégration du virus : Tat, Rev, les protéines de la nucléocapside et de la capside, et la protéase. En ce qui concerne les trois premiers, la mise au point d'inhibiteurs reste encore du domaine de l'hypothèse. Un produit issu du criblage systématique et supposé avoir une action anti-Tat pourrait cependant apparaître à relativement brève échéance en expérimentation humaine. Les inhibiteurs de Tat devraient entraîner une inhibition de la synthèse des ARN viraux. Les inhibiteurs de Rev quant à eux, devraient empêcher le passage dans le cytoplasme des grands messagers viraux et, par conséquent, la synthèse des protéines de structure du virion. Ceux de la nucléocapside devraient bloquer tout à la fois la dimérisation du génome viral (indispensable à la transcription inverse, donc à l'infectiosité des particules), et l'encapsidation, aboutissant à des particules vides d'ARN. De très nombreux travaux sont consacrés actuellement à ces protéines, dont la structure tridi- mensionnelle reste malheureusement très incomplètement connue. Quant à l'idée d'inhiber l'infectiosité de virus par des inhibiteurs de la protéine capsidiale dont les structures répétitives sont probablement essentielles pour assurer la formation de la coque interne principale du virus (la capside), elle reste très théorique [20]. Paradoxalement, la structure de cette protéine qui est l'une des plus importantes quantitativement dans le virion, est très mal connue. La mise au point d'inhibiteurs de la formation de la capside est une voie actuellement très peu explorée.

Les inhibiteurs de protéase sont, au contraire, des réalités qui arrivent au stade de l'expérimentation humaine. Des inhibiteurs peptidiques et surtout non peptidiques, dont on peut attendre une beaucoup plus grande efficacité en raison de leur stabilité, ont été mis au point [21-23]. Certains d'entre eux sont les résultats spectaculaires des travaux de modélisation moléculaire [23]. Ils atteignent des efficacités in vitro de l'ordre du nanomolaire mais on sait encore peu de choses de leur toxicité et de leur biodisponibilité. Ce groupe de médicaments provoque in vitro, la formation de particules immatures, incapables d'infecter des cellules parce que les grands précurseurs protéiques des produits des gènes gag et pol ne sont pas clivés. Cette piste est actuellement l'une des plus prometteuses pour l'avenir, à brève échéance, de la thérapeutique dirigée contre les lentivirus humains. Reste à démontrer s'ils sont actifs et bien supportés chez le malade.

Les oligonucléotides sens, antisens et les ribozymes

La thérapeutique antivirale, comme celle du cancer suscite un nombre considérable de recherches dont le but est de mettre au point des oligonucléotides à activité médicamenteuse [24]. Ces " anti-acides nucléiques " pourraient en théorie agir de plusieurs façons : en tant que neutralisants des ARN messagers et inhibiteurs de la traduction dans le cytoplasme, en tant que bloqueurs de l'épissage ou de la maturation des messagers dans le noyau, en tant que destructeurs d'ARN viraux, surtout lorsqu'il s'agit de ribozymes, ou 
encore, en tant que compétiteurs de produits d'activation, lorsqu'il s'agit d'oligonucléotides " sens " et non plus " antisens ". Leur utilisation se heurte à des difficultés majeures : leur sensibilité aux nucléases, qui semble en partie résolue actuellement, notamment par l'utilisation de nucléotides $\alpha$ et de phosphorothioates $[25,26]$, et leur pénétration cellulaire pour laquelle de nombreuses études de ciblage et de vectorisation sont en cours. Ce dernier obstacle reste difficile à franchir. Le maniement des oligonucléotides comme agent thérapeutique comporte encore d'autres inconnus dont la plus importante concerne les possibilités d'atteindre des concentrations intracellullaires qui seraient nécessaires, si bien que l'efficacité que l'on peut en attendre, en dehors de systèmes très particuliers, demeure incertaine. Nous ne saurons vraisemblablement que dans quelques années s'il existe un véritable avenir thérapeutique pour les oligonucléotides.

Les autres inhibiteurs de la réplication virale

Deux autres types de médicaments sont en principe susceptibles d'agir sur des cellules déjà infectées: les interférons et les inhibiteurs de glycosidase. Le mode d'action exact des interférons sur la réplication des lentivirus est mal connu : ils interviennent probablement à plusieurs étapes et plus particulièrement lors de la formation du bourgeon viral à la membrane cellulaire [27]. Malgré une certaine efficacité in vitro, les interférons utilisés in vivo se sont avérés décevants, même à doses relativement élevées. Cependant, leur utilisation en association avec d'autres médicaments agissant à d'autres stades de la réplication et susceptibles, éventuellement, d'en potentialiser l'action reste toujours justifiée. Ainsi des essais sont actuellement en cours pour tester l'efficacité de la coadministration d'AZT ou de ddC et d'interféron $\alpha$.

Les inhibiteurs de glycosidase dont le modèle initial est la castanospermine [28], semblent dotés d'une efficacité limitée exigeant des concentrations cellulaires difficilement accessibles in vivo. Ils interviendraient en modifiant la glycosylation de l'enveloppe virale et pourraient bloquer la réplication $\mathrm{m} / \mathrm{s} n^{\circ} 8$, vol. 7 , octobre 91 par deux mécanismes: d'une part, en limitant la sortie des particules de la cellule productrice, d'autre part, en diminuant l'infectiosité des particules filles. Leur utilisation reste pour le moment très théorique et n'a pas d'application prévisible à court terme en thérapeutique humaine.

\section{Utilisation d'autres protéines virales comme cibles thérapeutiques}

Les lentivirus humains et simiens sont caractérisés par l'expression d'une série de protéines que l'on ne rencontre pas dans les autres rétrovirus et dont nous ignorons pour l'essentiel la fonction. On soupçonne aujourd'hui que $\mathrm{Vpu}$ joue un rôle (exclusivement dans le cas du HIV-1) dans l'assemblage des particules virales et leur sortie de la cellule. Les mutants $\mathrm{Vpu}$ - produisent très peu de particules infectieuses. On peut donc imaginer en théorie que des inhibiteurs de Vpu pourraient aboutir au même résultat. De même, la protéine structurale $\mathrm{Vpr}$ semble intervenir dans l'activation de gènes cellulaires, très tôt après la pénétration du virus dans la cellule et joue peut-être un rôle à la phase initiale de l'infection. L'idée d'inhibiteurs de Vpr, et $a$ fortiori celle d'inhibiteurs de Vif et de Nef dont les fonctions sont encore très contestées restent pour le moment complètement théorique.

\section{Médicaments susceptibles de détruire électivement les cellules infectées}

De tels médicaments auraient en somme une action analogue à celle que l'on peut prêter naturellement aux lymphocytes $T$ tueurs qui sont présents et actifs en grande quantité chez les sujets porteurs du HIV. La destruction des cellules infectées aboutit, ipso facto, à une diminution de la production virale, tout particulièrement si elle peut avoir lieu à un stade précoce de la réplication, avant la production de particules complètes. Deux types d'approches sont théoriquement possibles :

- L'utilisation de médicaments qui seraient électivement toxiques pour les cellules infectées tout en respec- tant l'intégrité des cellules saines. C'est ce que certains ont cru devoir trouver dans l'emploi de la trichosantine ou GLQ223, extraite d'un concombre chinois [29]. Cette substance est en fait un poison des protéines ribosomales, particulièrement actif sur les cellules productrices de virus mais également très toxique sur la cellule saine. Les essais préliminaires qui ont été réalisés chez l'homme révèlent une toxicité importante, notamment encéphalique, et une action antivirale pour le moment incertaine. La plus extrême prudence semble indiquée dans l'emploi de tels médicaments.

- La deuxième possibilité consiste à cibler les cellules infectées et à y introduire une toxine grâce à la reconnaissance d'un marqueur de surface de ces cellules. Les immunotoxines, associant une toxine comme celle de la ricine ou du bacille diphtérique à une immunoglobuline sont une première solution. Une autre solution consisterait à utiliser une toxine couplée à une molécule CD4 [33]. Dans l'un et l'autre cas, le produit pourrait reconnaître à la surface cellulaire, la glycoprotéine d'enveloppe du virus et, injectant la toxine, détruire les cellules en cours de synthèse virale. Outre un maniement encore très incertain, ces conceptions se heurtent au fait que les cellules reconnues sont justement déjà en phase relativement avancée de production de particules virales puisqu'elles doivent exprimer la gp120 pour devenir la cible de ces drogues. Elles seraient donc le plus souvent rapidement détruites par le virus lui-même. Il serait plus intéressant de pouvoir identifier les cellules à un stade plus précoce, avant l'apparition de la réplication, mais nous ne connaissons pas de marqueurs de surface caractéristique de cette phase de réplication. Cette approche reste donc pour le moment expérimentale, mais elle mérite d'être poursuivie.

\section{Thérapie génique : immunisation intracellulaire et toxi-génétique}

Cette revue des approches médicamenteuses antivirales montre que 


\section{RÉFÉRENCES}

14. Karpas A, Hill F, Youle M, et al. Effect of passive immunization in patients with acquired immunodeficiency syndromerelated complex and acquired immunodeficiency syndrome. Proc Natl Acad Sci USA 1988 ; 85 : 9234-7.

15. Abrams DI, Kuno $\mathrm{S}$, Wong $\mathrm{R}$, Ueno R. Oral dextran sulfate UA001 in the treatment of the acquired immunodeficiency syndrome (AIDS) and AIDS related complex. Ann Int Med 1989 ; 110 : 183-8.

16. Clapham PR, Weber JN, Whitby D, et al. Soluble CD4 blocks the infectivity of diverse strains of HIV and SIV for T cells and monocytes but not for brain and muscle cells. Nature 1989 ; 337 : 368-70.

17. Capon DJ, Chamow SM, Mordenti J, et al. Designing CD4 immunoadhesins for AIDS therapy. Nature $1989 ; 337: 525-31$.

18. Byrn RA, Mordenti J, Lucas C, et al. Biological poperties of a CD4 immunoadhesin. Nature 1990 ; 344 : 667-70.

19. Ward RH, Capon DJ, Jett CM, et al. Prevention of HIV-1 IIIB infection in chimpanzees by CD4 immunoadhesin. Nature $1991 ; 352$ : 434-8.

20. Rossman MG. Antiviral agents targeted to interact with viral capsid proteins and a possible application to human immunodeficiency virus. Proc Natl Acad Sci USA 1988 ; 85 : 4625-7.

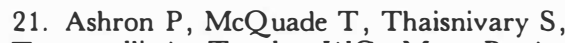
Tomasselli A, Tarpley WG, Moss B. An inhibitor of the protease blocks maturation of human and simian immunodeficiency viruses and spread of infection. Proc Natl Acad Sci USA 1990 ; 87 : 7472-6.

22. McQuade TJ, Tomasselli AG, Liv L, et al. A synthetic HIV-1 protease inhibitor with antiviral activity arrest HIV-like particle maturation. Science $1990 ; 247$; 454-6.

23. Roberts NA, Martin JA, Kinchington $\mathrm{D}$, et al. Rational design of peptide based HIV proteinase inhibition. Science $1990 ; 248$ : 358-64.

24. Hélène $C$, Thuong $N$. Le contrôle artificiel de l'expression des gènes. Pour la Science $1990 ; 7$ : 38-48.

25. Agrawal S, Ikenchi T, Sun D, et al. Inhibition of human immunodeficiency virus in early infected and chronically infected cells by antigens oligonucleotides and their phosphorothioate analogues. Proc Natl Acad Sci USA 1989 ; 86 : 7790-4. malgré l'existence des nucléosides inhibiteurs de la transcriptase réverse virale et de leurs analogues, seules molécules ayant une relative efficacité, et en dépit de nouvelles pistes prometteuses [31-35, Tableau $I$, p. 837, la synthèse de molécules antivirales véritablement efficaces risque de ne pas être rapide. L'importance de l'enjeu justifie donc de se tourner vers des stratégies moins conventionnelles telle la thérapie génique [36]. Certes, l'idée de protéger un individu contre une infection virale en modifiant le génome de ses cellules afin de les rendre résistantes, peut sembler inadaptée si l'on raisonne en terme d'infections virales aiguës. En revanche, comme le suggérait récemment $\mathrm{D}$. Baltimore [37], divers éléments rendent cette approche envisageable dans le cas d'une infection par le HIV. En effet cette infection induit une maladie chronique qui, en moyenne, n'aboutit à l'explosion du syndrome d'immunodéficience qu'en dix ans ou plus. Au cours de cette longue latence, le répertoire des cellules $\mathrm{T}$ est progressivement détruit et l'on peut donc imaginer qu'une thérapie génique pourrait au moins sauver une partie des cellules immunitaires. Cela ne permettrait pas de régler tous les problèmes qui surviennent au cours de l'infection, notamment l'atteinte du système nerveux central ou le développement des lymphomes, mais la thérapie génique pourrait limiter l'importance de l'immunodéficience qui est finalement à l'origine du décès de la majorité des patients. Par ailleurs, l'approche se trouve facilitée par le fait que le virus atteint les cellules $\mathrm{T}$ et les monocytes qui sont parmi les cellules les mieux adaptées aux techniques de transfert de gènes. Les cibles possibles d'une thérapie génique sont celles précédemment identifiées pour la thérapie médicamenteuse, mais le principe est bien différent. Il consiste en l'apport d'une séquence d'ADN capable de contrôler la synthèse d'une protéine, ou d'un ARN qui seront les molécules actives. On peut concevoir deux types d'approches: l'une basée sur le concept d'immunisation intracellulaire, c'est-à-dire sur l'introduction dans les cellules $\mathrm{T}$ de séquences d'ADN codant pour des molécules capables d'inhiber le cycle viral ; la seconde repose sur l'introduction de gènes dont l'expression sera toxique pour les cellules infectées par le HIV.

L'immunisation intracellulaire. Le concept a été introduit par D. Baltimore [37] comme une application au traitement des maladies virales de l'inactivation fonctionnelle de gènes par mutations négatives dominantes [38-41]. Il pourrait être appliqué au traitement de personnes déjà infectées, dans le but de rendre une partie de leurs lymphocytes et de leurs macrophages résistants à l'infection. Cela induirait alors véritablement un processus d'immunisation non immunologique permettant à une partie du répertoire T d'échapper à la destruction induite par le virus. Aussi longtemps qu'une greffe autologue de précurseurs hématopoiétiques modifiés s'avérera nécessaire, et qu'elle sera accompagnée de traitements préalables par des cytotoxiques ou par irradiation pour laisser place aux précurseurs réintroduits, ce traitement restera cependant inacceptable à titre préventif et même, peut-être, chez les patients récemment infectés. Néanmoins, il n'est pas exclu que l'utilisation de nouvelles stratégies de transfert à l'aide de virus recombinants directement injectables puisse modifier les données du problème. Ainsi, des rétrovirus ou adénovirus recombinants qui peuvent infecter les lymphocytes $\mathrm{T}$ et qui ne provoquent pas de maladies graves chez l'homme, pourraient être utilisés. De tels vecteurs pourraient être employés pour limiter les conséquences de l'infection dans les phases avancées de la maladie. On peut imaginer, en théorie, de les utiliser en traitement curatif plus précoce, avant l'apparition de l'immunodéficience, si tant est que ces vecteurs aient démontré leur inocuité. L'utilisation à visée vaccinale de vecteurs dont l'ADN s'intègre dans le génome de l'hôte, pouvant ainsi provoquer des mutations insertionnelles délétères, reste plus problématique.

Comme l'ont proposé Herskowitz [38] et Baltimore [37], au moins deux types de protéines virales pourraient être inhibées par l'introduction de gènes mutants transdominants dans les cellules $T$ : des gènes codant 
Tableau I

THÉRAPIES ANTIVIRALES: APPROCHES CONVENTIONNELLES

\begin{tabular}{|c|c|c|c|}
\hline Niveau de blocage & Cible & Produit & Efficacité \\
\hline $\begin{array}{l}\text { Interaction virus/récepteur cellulaire } \\
\text { Fusion de l'enveloppe virale avec la membrane } \\
\text { cellulaire }\end{array}$ & $\begin{array}{l}\text { Gp120 } \\
\text { Gp120 } \\
\text { Gp41 }\end{array}$ & $\begin{array}{l}\text { CD4 soluble } \\
\text { Polysaccharides } \\
\text { Anticorps } \\
\text { Anticorps }\end{array}$ & $\begin{array}{l}\text { Très faible } \\
\text { Très faible }\end{array}$ \\
\hline Transcription inverse & Transcriptase inverse & $\begin{array}{c}\text { Nucléosides } A Z T \\
\text { TIBO }\end{array}$ & $\begin{array}{l}\text { Réelle } \\
\text { mais } \\
\text { limitée }\end{array}$ \\
\hline $\begin{array}{l}\text { Transport de l'ADN viral au noyau } \\
\text { Intégration du provirus } \\
\text { Provirus } \\
\text { Transcription } \\
\text { Traduction } \\
\text { Assemblage du virion } \\
\text { Bourgeonnement viral } \\
\text { Encapsidation et dimérisation des ARN viraux } \\
\text { Glycosylation de l'enveloppe } \\
\text { Maturation du virus }\end{array}$ & $\begin{array}{c}? \\
\text { Intégrase } \\
\text { Provirus } \\
\text { Tat } \\
\text { ADN viral } \\
\text { ARNm } \\
\text { p24 } \\
? \\
\text { Vpu } \\
\text { Nucléocapside P15, P7 } \\
\text { Glycosidases cellulaires } \\
\text { Protéase virale }\end{array}$ & $\begin{array}{c}? \\
? \\
\text { Antisens } \\
\text { Inhibiteur de Tat } \\
\text { Antisens } \\
\text { Antisens } \\
? \\
\text { Interféron } \\
? \\
? \\
\text { Castanospermine } \\
\text { Inhibiteur des protéases }\end{array}$ & $\begin{array}{c}- \\
\bar{?} \\
? \\
? \\
? \\
? \\
? \\
\text { Faible } \\
- \\
- \\
? \\
?\end{array}$ \\
\hline & Cellules infectées & Immunotoxines & \\
\hline
\end{tabular}

Tableau II

THÉRAPIES GÉNIQUES ANTIVIRALES POTENTIELLES

\begin{tabular}{|l|c|c|}
\hline \multicolumn{1}{|c|}{ Niveau de blocage } & Cible & Transgène codant pour \\
\hline Interaction virus/récepteur cellulaire & Gp120 & CD4 soluble \\
\hline Formation de syncitia & Gp120 & $\begin{array}{c}\text { CD4 piégé dans le réticulum } \\
\text { endoplasmique }\end{array}$ \\
\hline Suicide des cellules infectées & Cellule infectée & Toxine conditionnelle \\
\hline Assemblage du virion & Protéines de structure multimériques & Mutants transdominants \\
\hline Maturation et transport des ARN viraux & Rev & Mutants transdominants \\
\hline Transcription & Tat & Mutants transdominants \\
\hline Traduction des protéines virales & Messagers viraux & Antisens ribozyme \\
\hline
\end{tabular}




\section{RÉFÉRENCES}

26. Buck HM, Koole LH, Van Genderen MHP. Phosphate methylated DNA aimed at HIV-1 RNA loops and integrated DNA inhibits viral infectivity. Science $1990 ; 248$ : 208-12.

27. Poli G, Orenstein JM, Kinter A, Polks TM, Fauci AS. Interferon alpha but not AZT suppresses HIV expression in chronically infected cell lines. Science 1989 ; 244: 575-7.

28. Walker BD, Kowalski M, Gohwc, et al. Inhibition of human immunodeficiency virus syncytium formation and virus replication by castanospermine. Proc Natl Acad Sci USA $1987 ; 84: 8120-4$.

29. McGrathe MS, Hwang KM, Caldwell SE, et al. GLQ223 an inhibitor of human immunodeficiency virus replication in acutely and chronically infected cells of lymphocyte and mononuclear phagocyte lineage. Proc Natl Acad Sci USA 1989 ; 86 : 2844-8.

30. Aufray C, Piatier-Tonneau D, Kroemer G. CD4-targeted immune intervention : a strategy for the therapy of AIDS and autoimmune disease. Trends Biochem Tech $1991 ; 9$ : 124-31.

31. Mitsuya H, Yarchoan R, Kageyama S, Broder S. Targeted therapy of human immunodeficiency virus-related diseases. FASEB J 1991 ; 5 : 2369-81.

32. Broder S, Mitsuya H, Yarchoan R, Pavlakis N. Antiretroviral therapy in AIDS. Ann Inter Med 1990; 113 : 604-18.

33. Mitsuya H, Yarchoan R, Broder S. Molecular targets for AIDS therapy. Science $1991 ; 249$ : 1533-44

34. Darlix JL. Nouvelles perspectives en chimiothérapies antirétrovirales. médecine/sciences $1989 ; 5: 213-9$

35. Johnson RP, Schooley RT. Update on antiretroviral agents other than zidovudine. AIDS 1989 ; 3 (suppl 1) : S145-51.

36. Kahn A, Briand $P$. Thérapies géniques : espoirs et limites. médecine/sciences $1991 ; 7$ : 705-14.
A

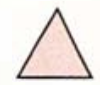

Protéine normale

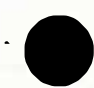

Protéine défectueuse codée par le transgène

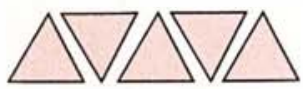

Structure normale

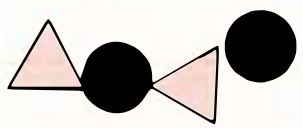

Structure instable

B

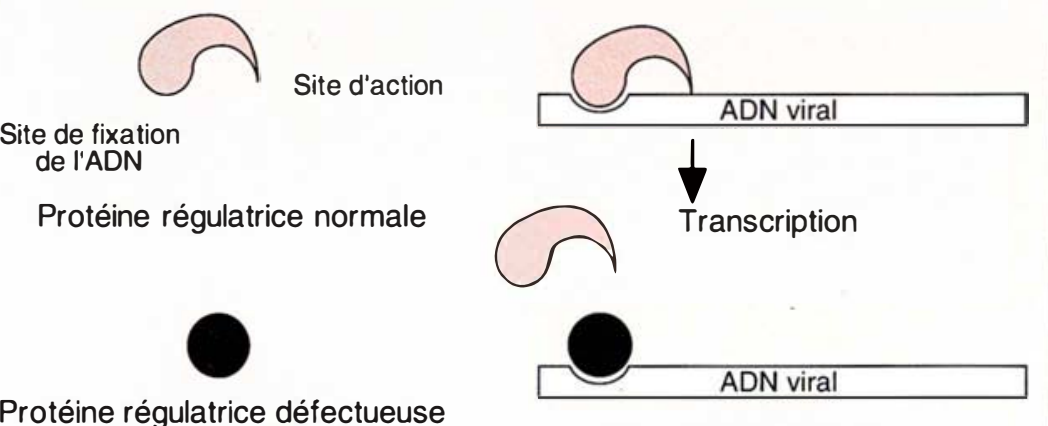

Protéine régulatrice défectueuse codée par le transgène

Pas de transcription

C

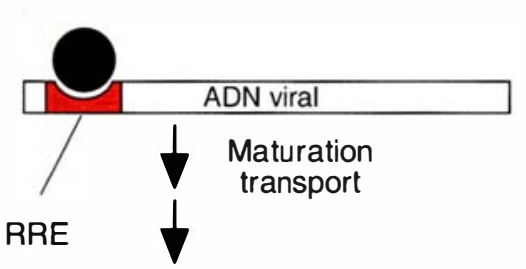

ARN "leurre" transgénique

Traduction

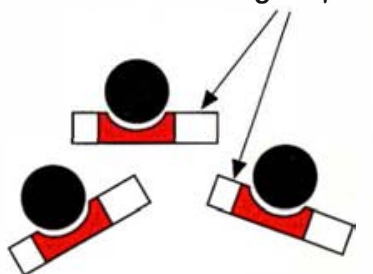

ADN viral

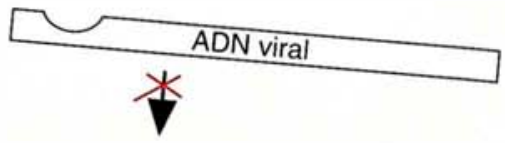

D

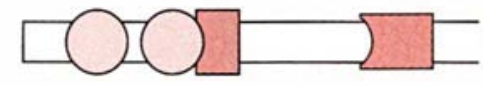

Membrane cellulaire

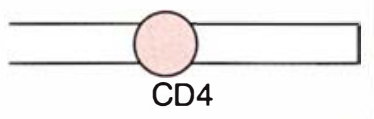

Réticulum

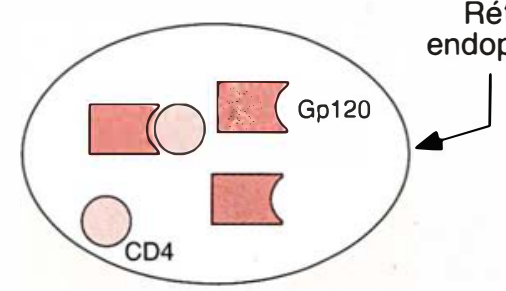

Cellule CD4+ infectée non transgénique

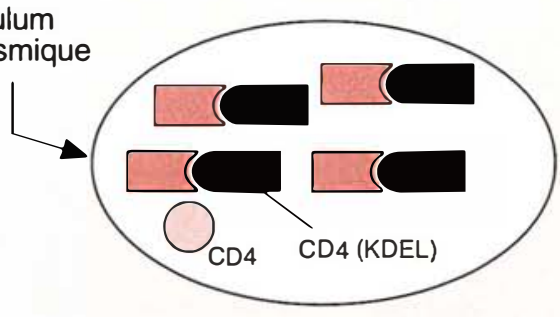

Cellule CD4+ infectée transgénique synthétisant CD4 KDEL 
pour des protéines structurales oligo ou multimériques (figure $3 A, p .838$ ) et des gènes codant pour des protéines régulatrices polymériques ou monomériques (figure $3 B, 3 C, p$. 838). Après la démonstration initiale par Friedman et al. [39] que l'expression d'une protéine virale transactivatrice tronquée du virus Herpes simplex de type 1 (HSV-1) pouvait empêcher l'infection lytique des cellules traitées par le HSV, deux groupes ont rapporté des résultats préliminaires suggérant une application possible au HIV-1. Malim et al. [40] ont montré qu'un mutant du gène rev pouvait inhiber de façon transdominante la fonction normale de la protéine Rev, à savoir le transport des ARNm viraux du noyau vers le cytoplasme, dans un système d'expression transitoire. La cotransfection par le gène mutant, induit un effet inhibiteur marqué sur l'expression des gènes viraux. Les résultats de ces expériences peuvent être interprétés comme la manifestation d'une compétition exercée par la protéine mutante qui aurait gardé la possibilité de s'associer à l'ARN viral, mais aurait perdu son site fonctionnel, comme cela avait été suggéré dans le modèle de la protéine VP16 modifiée du virus herpes [39]. Cet effet n'a cependant pas été testé dans des cellules infectées par le HIV-1 et nous ignorons par conséquent si le niveau de protéine Rev mutante produite serait suffisant pour avoir un effet protec- teur anti-HIV. De la même façon, Green et al. [41] ont montré que dans des cellules Hela dans lesquelles un gène rapporteur chloramphénicol acétyl transférase (CAT) contrôlé par les séquences régulatrices (LTR) du HIV avait été introduit, des mutants de Tat pouvaient inhiber par compétition la transactivation du LTR induite par la protéine Tat normale. Là-encore, nous ignorons si un tel gène serait efficace dans des cellules infectées, mais l'ensemble de ces expériences suggère que l'introduction de gènes codant pour des protéines régulatrices mutées pourrait avoir, comme dans le cas du virus Herpes, une réelle efficacité antivirale.

Une autre possibilité serait l'introduction de gènes viraux codant pour des protéines structurales multimériques mutées. Comme l'ont proposé Trono et al. [42], une expression modérée de telles protéines pourrait avoir un puissant effet inhibiteur. Cette idée a été appliquée par ces auteurs pour bloquer la réplication du HIV après transfection des gènes gag p17 et p24 mutés. Ils ont, en outre, montré que dans des cellules exprimant de façon constitutive la protéine de capside Gag p24, le virus avait une capacité réplicative affaiblie. Il est possible de concevoir que la réplication virale puisse être inhibée à différent niveau incluant l'assemblage, le relargage, la stabilité des virions, voire même la pénétra-

4 Figure 3. Exemples d'immunisation intracellulaire antivirale. 3A : transgène " mutant transdominant " codant pour une protéine structurale multimérique. L'association des monomères normaux codés par le gène viral et des monomères mutés codés par le transgène entraine la formation de structures instables incompatibles avec la production d'un virion infectieux. 3B : transgène " mutant transdominant " codant pour une protéine régulatrice monomérique. 3C : transgène codant pour un "leurre ". La protéine virale Rev indispensable au transport des divers messagers du noyau dans le cytoplasme agit en reconnaissant une séquence RRE sur les messagers (rev responsive element). La production massive de séquences cibles RRE pourrait détourner la protéine Rev de son site d'action normal et empêcher l'expression des protéines virales. 3D : transgène codant pour un récepteur CD4 muté comportant un signal de rétention dans le réticulum endoplasmique. La protéine virale Gp 120 synthétisée dans les cellules infectées exprimant le transgène, s'associe à la molécule CD4 modifiée et se trouve piégée dans le réticulum. La formation des syncitia, liée à la présence de molécules Gp120 à la surface des cellules infectées, ne peut avoir lieu.

$\mathrm{m} / \mathrm{s} n^{\circ} 8$, vol. 7 , oclobre 91 tion ou la décapsidation dans une seconde génération de cellules infectées. Ces résultats encourageants basés sur une immunisation intracellulaire, doivent maintenant être complétés par des études in vivo.

La synthèse intracellulaire d'un récepteur viral modifié a été proposée par Buonocore et Rose [43] pour tenter de contrecarrer la réplication virale dans les cellules infectées (figure 3D). Ces auteurs ont utilisé un gène CD4 muté codant pour une forme soluble modifiée du CD4 comportant un signal de rétention dans le réticulum endoplasmique (séquence KDEL). Dans les cellules exprimant ce mutant, la protéine d'enveloppe gp1 20 qui s'associe au CD4 se trouve piégée dans le réticulum et n'apparaît donc pas à la surface des cellules. De plus, la fusion avec d'autres cellules exprimant le CD4 à leur surface qui provoque nombre des effets cytopathiques du virus, est inhibée. Il est à remarquer qu'étant donné l'extrême conservation du domaine d'association de la protéine gp120 au CD4, de nombreux isolats pourraient être inhibés par la même protéine mutante.

L'utilisation d'oligonucléotides, de messagers antisens ou de ribozymes et les problèmes que posent leur utilisation ont déjà été évoqués dans le chapitre des nouveaux médicaments potentiels. Ces problèmes incluent : (a) la stabilité des oligonucléotides; (b) le ciblage, la pénétration et l'accessibilité au compartiment cellulaire adapté ; (c) l'obtention dans les cellules infectées de concentrations suffisantes des molécules choisies, ce qui semble l'un des points cruciaux, particulièrement en ce qui concerne l'utilisation d'antimessagers. Certains de ces problèmes pourraient être résolus par l'obtention d'une expression stable de gènes codant pour des messagers viraux antisens dans des cellules souches de la moelle osseuse, qui pourraient ensuite être greffées chez le donneur autologue. Il s'agirait là-encore d'une immunisation intracellulaire, mais au niveau de l'ARN. Il y a encore peu de résultats en ce domaine, néanmoins, Sarver et al. [44] ont rapporté que la transfection de cellules Hela-CD4 + avec un gène codant pour un ribozyme dirigé contre la protéine 


\section{RÉFÉRENCES}

37. Baltimore D. Intracellular immunization. Nature 1988 ; 335 : 395-6.

38. Herskowitz I. Functional inactivation of genes dominant by negative mutations. Nalure 1987 ; 329 : 219-22.

39. Friedman AD, Triezenberg SJ, McKnight SL. Expression of a truncated viral transcriptor selectively impedes lytic infection by its cognate virus. Nature 1988 ; $335: 452-5$.

40. Malim $\mathrm{MH}$, Bölinlein S, Hauber J, Cullen BR. Functional dissection of the HIV-1 Rev transactivator. Derivation of a trans-dominant repressor of Rev function. Cell 1989 ; 58 : 205-14.

41. Green M, Ishino M, Loewarstein PM Mutational analysis of HIV-1 Tat minimal domain peptides : identification of transdominants mutants that suppress HIV-LTR driven gene expression. Cell 1989 ; 58 : 215-23.

42. Trono D, Feinberg MB, Baltimore D. HIV-1 Gag mutants can dominantly interfere with the replication of the wild type virus. Cell 1989 ; 59 : 113-20.

43. Buenocore L, Rose JK. Prevention of HIV-1 glycoprotein transport by soluble CD4 retained in the endoplasmic reticulum. Nalure 1990 ; 345 : 627-8.

44. Sarver N, Cantin EM, Chang PS, el al. Ribozymes as potential anti-HIV-1 therapeutic agents. Science $1990 ; 247$ : 1222-5.

45. Sullenger BA, Gallardo HF Ungers GE, Gibbon E. Overexpression of TAR sequences renders cells resistant to human immunodeficiency virus replication of the wild type virus. Cell 63: 601-8.

46. Harrisson GS, Maxwell F, Long CJ, Rosen CA, Glode M, Maxwell IH. Activation of a diphteria toxin 1 gene expression of human immunodeficiency virus-1 Tat and Rev proteins in transfected cells. Hum Gene Ther 1991; 2 : 53-60.

47. Venkatesh LH, Avens MQ, Subramanian $T$, Chinnadurai G. Selective inhibition of toxicity to human cells expressing human immunodeficiency virus type 1 Tat by a conditionaly cytotoxic adenovirus vector.
Proc Nall Acad Sci USA 1990; 87 : 8746-50.
Gag, pouvait entraîner une réduction notable de l'expression des ARN Gag après infection par le virus HIV-1. Dans ces expériences, le niveau de la protéine p24 Gag était réduit d'un facteur 50 à 100 . De plus, la quan. tité d'ADN proviral était aussi réduite d'un facteur 100 suggérant la capacité du ribozyme non seulement de diminuer la production virale par des cellules infectées mais aussi de protéger la cellule contre une infection en clivant l'ARN viral dès sa pénétration. Ces résultats laissent donc espérer qu'une thérapie génique pourrait être réalisée non seulement avec des gènes mutants négatifs dominants, mais aussi avec des gènes codants pour des ribozymes ou des antisens.

Une approche voisine aurait recours à des ARN "sens". L'inhibition de la transactivation par la protéine Tat a déjà été évoquée comme cible possible de thérapie génique par production de protéines mutantes transdominantes. Une autre stratégie pourrait être de piéger la protéine $\mathrm{T}$ at par une séquence constituée de la séquence $A R N$ TAR qui est la cible naturelle de la transactivation des ARN viraux par la protéine Tat. Un gène codant pour un ARN " sens" TAR a ainsi été récemment transfecté dans des cellules CEM par Sullenger et al. [45]. En utilisant un vecteur rétroviral dans lequel une séquence hybride tARN-met-TAR était insérée dans les deux LTR, ces auteurs ont obtenus de très hauts niveaux d'expression de la séquence TAR et une inhibition de près de $99 \%$ de l'expression de la protéine de capside p24 après infection des cellules par le HIV. Là-encore, cette stratégie ne se heurterait pas à la variabilité du virus puisque les protéines Tat des virus HIV-1, HIV-2 ou SIV, interagissent toutes avec la séquence TAR du HIV-1.

La thérapie génique par toxigénétique. L'élimination sélective des cellules infectées par le HIV-1 est délicate et la production de molécules qui ne seraient toxiques que pour les cellules inf ectées reste problématique. Certes, les immunotoxines ou toxines associées à la molécule CD4 précédemment évoquées, pourront peut-être être utilisées mais leur spé- cificité comme leur efficacité in vivo, est loin d'être démontrée. Enfin, les marqueurs membranaires connus comme étant spécifiques des cellules infectées, spécialement les molécules Env, apparaissent tardivement à la surface cellulaire; par conséquent, seules des cellules largement engagées dans la production virale pourraient être éliminées. Une autre approche pourrait être basée sur la toxigénétique, c'est-à-dire une thérapie génique liée à l'expression conditionnelle, dépendante de la présence du virus, d'une toxine cellulaire. Il s'agirait en quelque sorte de la programmation d'un ordre de suicide donné aux cellules infectées. On peut pour ce faire, introduire dans des cellules CD4 + un gène hybride codant pour une toxine, placé sous le contrôle de séquences régulatrices virales (figure 4). Un tel gène devrait rester silencieux en l'absence de protéine transactivatrice. Tat induirait son expression et par conséquent le suicide des cellules infectées. Une sécurité supplémentaire limitant les risques d'élimination de cellules non infectées comportant des protéines cellulaires à activité de type Tat, pourrait être apportée en incluant dans le gène codant pour la toxine, une séquence de rétention nucléaire des messagers et une séquence cible de la protéine virale Rev (RRE) [46] (figure 4). Ainsi en l'absence de Tat et de Rev, la toxine ne pourrait pas être synthétisée. Une telle stratégie pourrait permettre de limiter considérablement la propagation du virus, en particulier chez les malades récemment infectés. En outre, l'introduction dans l'organisme de vecteurs viraux recombinants tels les adénovirus, permettant une thérapie génique directe sans passer par une greffe de moelle, pourrait rendre cette approche plus opérationnelle. Récemment, Venkatesh et al. [47], ont montré qu'il était possible de transférer à l'aide d'un adénovirus recombinant un gène de toxicité conditionnelle (le gène codant pour la thymidine kinase (TK) du virus herpès), sous contrôle du LTR du HIV-1 (LTRHIV/HSV-TK). Lorsque des cellules Hela exprimant la protéine Tat sont infectées par cet adénovirus recombinant LTR-HIV/HSV-TK et mises en présence de gancyclovir, elles 


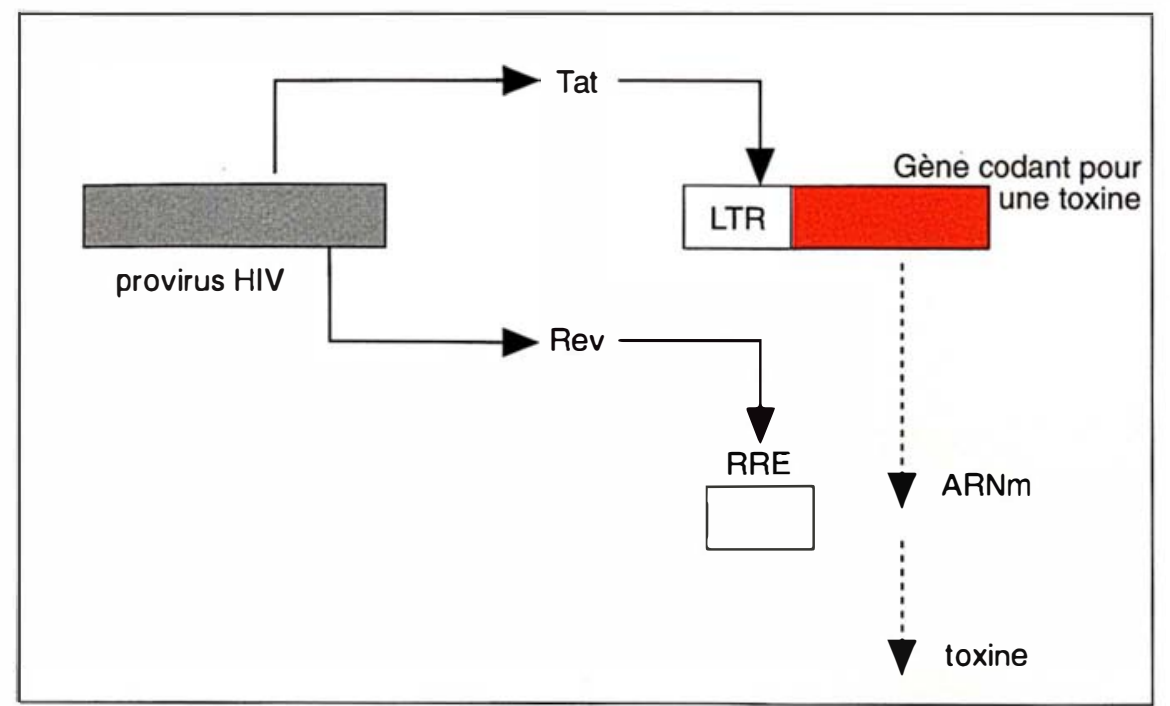

Figure 4. La thérapie génique antivirale par toxigénétique repose sur l'expression conditionnelle, dépendante de la présence du virus, d'une toxine. Le gène codant pour la toxine est placé sous la dépendance des séquences régulatrices virales (LTR du virus HIV-1) dont l'activité nécessite la présence de la protéine transactivatrice virale Tat. En principe, la toxine ne sera exprimée que dans les cellules infectées et les détruira donc spécifiquement. II existe cependant, dans certains types de cellules non infectées, des protéines capables de transactiver le LTR viral. Afin d'éviter une expression de la toxine dans ces cellules on peut introduire dans le transgène des séquences qui conditionnent l'expression de la toxine à la présence d'une seconde protéine virale : la protéine Rev. II s'agit d'une séquence de rétention nucléaire et du RRE (Rev responsive element). Ainsi, dans une cellule non infectée mais possédant une protéine de type Tat, le messager est transcrit mais reste dans le noyau et n'est pas traduit. En revanche, dans une cellule infectée la présence de Tat et de Rev aboutit à la production de la toxine et la cellule est détruite.

métabolisent cet analogue nucléotidique et en meurent dans $98 \%$ des cas. Il reste cependant à résoudre divers problèmes : (a) des résultats moins spectaculaires ont été obtenus dans d'autres types cellulaires dont des cellules T ; (b) une toxicité notable a été observée vis-à-vis de cellules non infectées ; (c) nous ignorons enfin si les cellules infectées pourraient être éliminées avant la production de particules virales. Néanmoins, ces résultats suggèrent qu'un adénovirus pourrait permettre le transfert et l'expression d'une toxine, entraînant la destruction de cellules infectées, dès la production des messagers viraux précoces.

Les thérapies géniques du SIDA sont manifestement à un stade précoce de leur élaboration, mais les premiers résultats obtenus ex vivo montrent les potentialités d'une telle approche thérapeutique. Il reste que leur efficacité doit être maintenant testée in vivo sur des modèles murins, des macaques ou des chats infectés par des lentivirus. La réalité de leur application future sera aussi fonction du succès ou de l'échec des approches conventionnelles et restera probablement complémentaire de ces dernières. Si l'ampleur des enjeux justifient d'ajouter aux stratégies plus classiques d'élaboration thérapeutique anti-SIDA cette voie potentielle qu'est la thérapie génique, il faut néanmoins souligner que son application à grande échelle dans les pays, et particulièrement en Afrique où des millions de personnes sont concernées, pourrait encore rester très longtemps une utopie

\section{Summary}

Therapy of AIDS : towards new drugs and strategies including gene therapy

Since the discovery of human immunodeficiency virus (HIV) as the primary cause of AIDS, a number of potential strategies for antiretroviral therapy of this disease have emerged. A classical approach, relying on random $e x$ vivo screening for potential antiHIV activity of number of molecules, has led to the identification of AZT as a therapeutic agent against HIV-1. It has been the first drug tested in individuals with AIDS and remains the only moleculc currently used. Other nucleoside type drugs and AZT derivatives are tested in the aim to found more active and less toxic molecules. Furthermore, enough insight has been gained into some of the virus structure proteins to provide basis for molecular design of antiviral drugs. Such an approach has been successfully used to obtain protease inhibitors that are active at least ex vivo. Nevertheless, despite the fact that all the stages of HIV-1 replicative cycle are identified and considered potential targets for therapeutic intervention, classical strategics have only provided antiviral drugs that remain poorly efficient. Even if improvements can be expected with the appearance of new families of molecules, the development of efficient antiviral therapy of AIDS is likely to be a very long story. It is therefore logical to consider other approaches such as gene therapy (intraccllular immunisation or toxi-genetics), cven though their eventual application would remain for a long time difficult at a large scale and even mythical in underdeveloped countries. 